\title{
Vingt ans après \\ La Rance, une expérience marémotrice
}

\section{Twenty years after : an experiment in tidal power}

\author{
P. Hillairet \\ Chef du Groupe mixte Exploitation Bretagne \\ Electricité de France
}

Toujours seule, unique au monde, contre vents mais avec les marées, elle fonctionne... Où en est la centrale marémotrice de la Rance en 1984 ?

En un bref raccourci, rappelons qu'en 1952, un Président de la République, Vincent Auriol, inaugurant Donzère Mondragon, annonçait l'aménagement prochain de l'estuaire de la Rance, qu'en janvier 1961 débutaient les travaux, que le $1^{\text {et }}$ août 1966 était couplé le premier groupe, que l'inauguration de l'ouvrage était présidée par un autre Président de la République le 26 novembre 1966 , mais que la mise en service de la $24^{e}$ et dernière machine n'avait lieu que le 4 décembre 1967.

Depuis, des milliards de $\mathrm{m}^{3}$ ont franchi l'ouvrage, environ $7000 \mathrm{~km}^{3}$, essayant d'user, d'éroder, d'oxyder ce que les hommes avaient osé construire dans cet estuaire. Qu'en est-il aujourd'hui de cette installation?

- les ouvrages de génie civil ont-ils résisté à l'assaut de la mer ?
- la corrosion marine a-t-elle dévoré les turbines ? - l'environnement a-t-il été perturbé par la présence insolite de ce pont barrage?

Autant de questions que beaucoup d'entre vous peuvent légitimement se poser, auxquelles répondent ceux qui ne savent pas et qui, dans la région, annoncent délibérément: "la Rance? elle s'envase et un seul groupe tourne"

Les mouettes, elles, pauvres volatiles intelligents, ne se trompent pas, qui s'alignent sur le couronnement, à l'amont ou a l'aval suivant le sens de fonctionnement et qui récupèrent à leur profit les poissons étourdis par leur passage forcé dans les pertuis.

Les constatations résumées dans cette communication vous permettront de vous faire une opinion, puisse-t-elle être satisfaisante.

\section{Ouvrages de génie civil}

\subsection{Caractéristiques des ouvrages}

Elles donnent, bien que connues, une idée de l'installation par leurs dimensions et la quantité des matériaux mis en œuvre.

De la rive gauche à la rive droite :

- une écluse de $13 \mathrm{~m}$ de largeur;

- l'usine de $390 \mathrm{~m}$ de longueur, $53 \mathrm{~m}$ de largeur et $33 \mathrm{~m}$ de hauteur;

- la digue monte en enrochement de $160 \mathrm{~m}$ de longueur;

- le barrage mobile de $115 \mathrm{~m}$ équipé de 6 pertuis de vannage de $15 \mathrm{~m} \times 10 \mathrm{~m}$

Le volume des bétons est de $350000 \mathrm{~m}^{3}$ pour 16000 tonnes d'acier et la surface des parements exposés à l'eau de mer est de $90000 \mathrm{~m}^{2}$.
C'est dire tout ce que le constructeur offrait à l'épreuve de la mer avec un ouvrage de cette importance et la complexité des formes qui le caractérise, loin de celles habituellement rencontrées en ces lieux : les longs quais d'amarrage des navires.

\subsection{Structures en béton armé}

Les expérimentations préalables - éprouvettes en situation - les précautions prises à la construction, traitement des rochers, lavage des sables, agrégats et ciments de qualité, pervibration soignée... - ont été tels qu'après 20 ans d'exposition à l'atmosphère marine le comporte- 


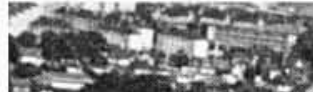

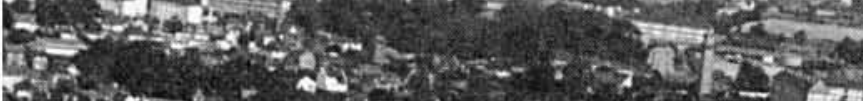

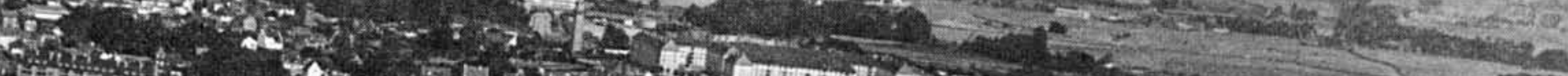

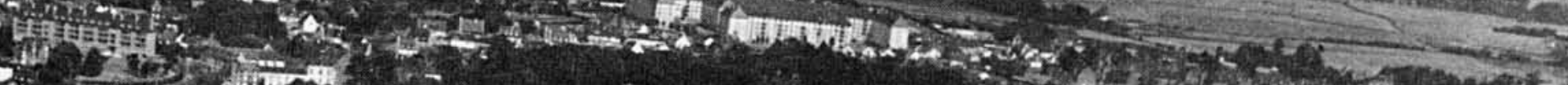

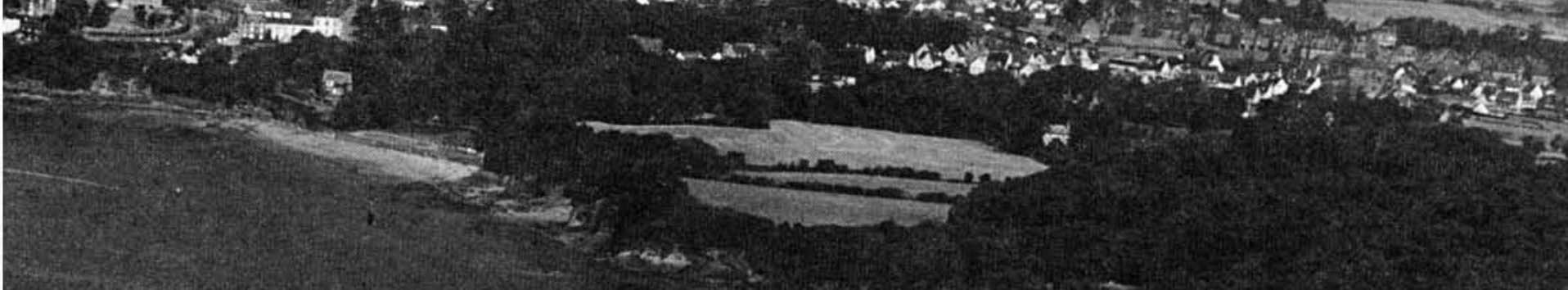
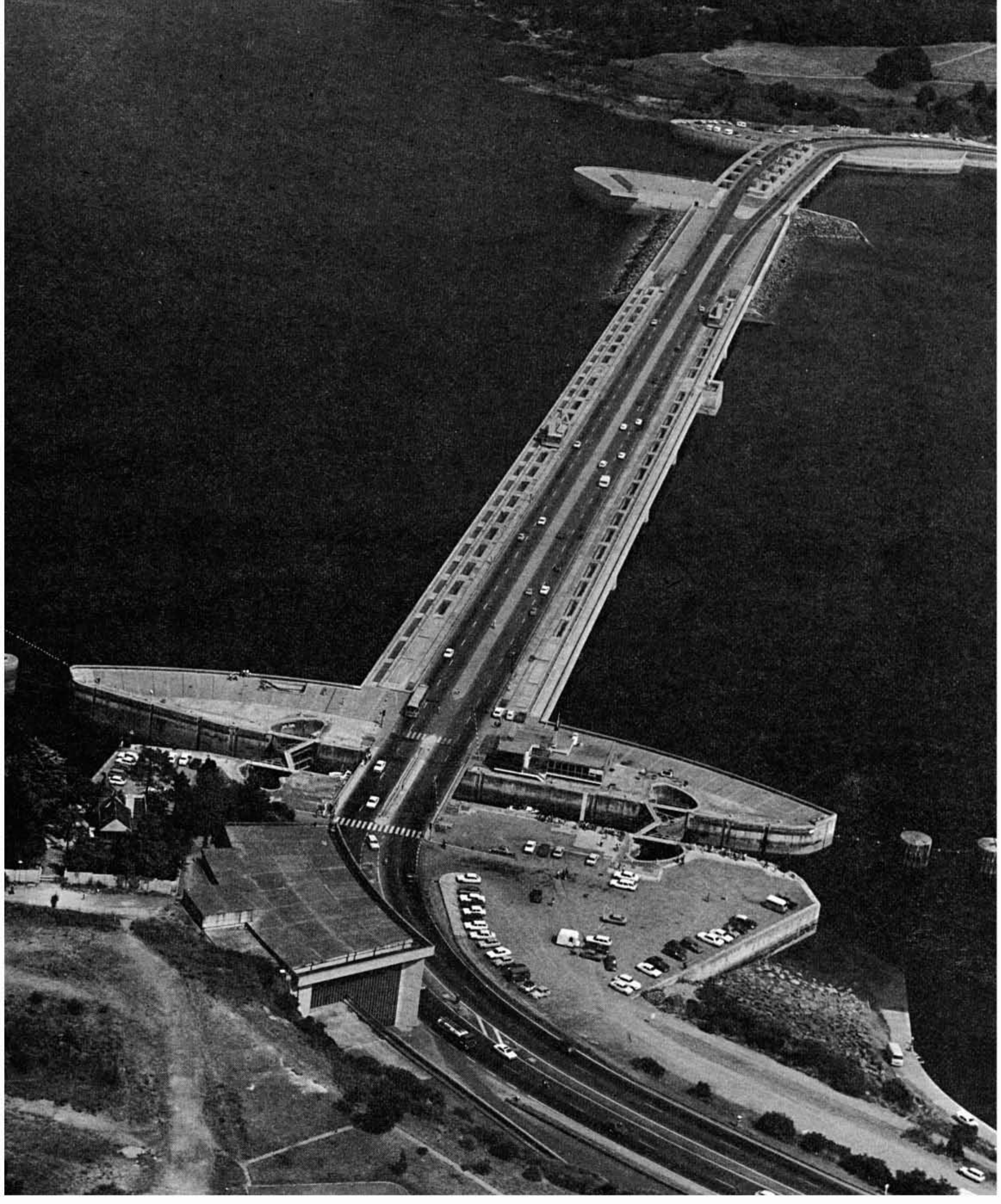
ment des ourages de Génie Civil est pratiquement parfait, l'aspect des parements impeccable :

a) Vous ne parcourrez pas les $700 \mathrm{~m}$ de l'ouvrage sans détecter une fissure, elle peut être de retrait, bouchée ou non par une formation de calcite, elle ne sera ni de tassement ni évolutive. Elles ont fait l'objet de traitements à l'origine, mais elles pourraient être assimilées aux quelques rides d'un ouvrage qui vieillit tout doucement.

Pourrez-vous également observer quelques dégradations superficielles inévitables dans la zone de marrage entre $8 \mathrm{~m}$ et $12 \mathrm{~m}$. Elles n'ont pas de conséquences dans l'immédiat, n'évoluent que très, très lentement et au rythme actuel ne créent aucun souci particulier. Quoiqu'il en soit, les matériaux actuels, résines époxy... permettraient de les traiter facilement et sans grand problème si on prend de surplus la précaution de faire ces travaux en mortes eaux.

b) On ne note d'autre part aucune déformation de l'ouvrage - qui se traduirait aussitôt par des contraintes mécaniques sur les vannes, les groupes, les rails de roulement des portiques... - , aucune décomposition du béton. Les seules parties traitées de l'ouvrage ont été quelques affouillements décelés par visite sous-marines et traités de même. Les affouillements restent toutefois de faible importance et ne menaceraient pas l'ouvrage s'ils n'étaient découverts que tardivement.

c) Monsieur Duhoux avait mentionné en 1972 l'oxydation des armatures du pont routier...

Il est bien évident que cette situation ne s'est pas améliorée, sans pour autant être catastrophique. Elle conduit cependant le Conseil Général de l'Ille et Vilaine à prévoir la réalisation de travaux d'un montant de l'ordre de $5000 \mathrm{KF}$, qui pourrait paraître élevé quand il ne s'agit que d'oxydation superficielle, mais la mise en place d'échaffaudages et l'environnement particulier dans lequel ces travaux seraient effectués justifient sans doute une telle dépense.

\subsection{Etanchéité de l'ouvrage}

La valeur des débits de fuite, de l'ordre de 5 litres par minute, son évolution ne sont que la preuve concrète de ce que constate le visiteur qui pénétrant dans la salle des machines n'aperçoit aucune trace d'humidité et est frappé, voire surpris, par l'aspect impeccable des murs amont et aval de l'ouvrage. Il en sera de même s'il descend dans les galeries à $-12 \mathrm{~m}$. C'est dire l'étanchéité tout à fait satisfaisante de l'ouvrage qui ne donne lieu à aucune préoccupation particulière mais n'exclut pas pour autant des visites et contrôles systématiques.

Quelques travaux d'injection ont été réalisés après la mise en eau entre 1968 et 1972 et d'autres en 1981 et 1982 par injection de résine acrylique polymérisable.

\subsection{Démoulage}

(moules, algues, coquillages...)

Sans que ces dépôts aient une incidence quelconque sur la bonne tenue des ouvrages - encore qu'ils pourraient en avoir sur la production - ils sont intéressants à noter car ils ont leur importance pour les exploitants. Il faut quand même savoir qu'à chaque vidange de pertuis pour intervention à l'extérieur d'un bulbe, la première et indispensable opération à faire, sous peine d'empoisonnement, est d'enlever les dépôts qui tapissent les bajoyers.

Ce nettoyage nécessite 30 hommes/jours de travail par groupe, coûte environ $25 \mathrm{KF}$ et représente environ 1000 $\mathrm{kg}$ de dépôt.

\section{Comportement du matériel électro-mécanique}

\subsection{Caractéristiques}

La Centrale est équipée de 24 groupes bulbes turboalternateurs constitués par :

- une turbine Kaplan : débit $275 \mathrm{~m}^{3} / \mathrm{s}$;

- un alternateur de $10 \mathrm{MW}$ : vitesse de rotation $93.75 \mathrm{tr} / \mathrm{mn}$, tension $3,5 \mathrm{kV}$, sous air surpressé à 2 bars.

Chaque "ensemble" de 4 groupes débite sur le tertiaire d'un transformateur de "bloc" $3.5 / 220 \mathrm{kV}$ de 80 MVA relié directement au réseau $220 \mathrm{kV}$ par câble à huile sous pression de 3,5 bars. IL existe donc 6 " ensembles" et 3 "blocs ", terminologie peut-être compliquée mais tellement familière aux exploitants de la Rance.

Les groupes de la Rance ont été construits pour un fonctionnement assez inhabituel qu'il est indispensable de rappeler si on veut avoir une bonne idée des contraintes électriques et mécaniques auxquelles sont soumises les machines.
Comme les cycles d'exploitation utilisent le pompage, ces groupes doivent pouvoir fonctionner en alternateur et en pompe, si l'on ajoute le fonctionnement en orifice utilisé pour accélérer les opérations de vidange et remplissage et qu'en plus on utilise la marche en double effet on obtient les 6 types de fonctionnement suivants :

- turbinage direct, pompe inverse, machine couplée, vidange du bassin,

- orifice direct, machine non couplée, vidange du bassin, - turbinage inverse, pompe directe, machine couplée, remplissage du bassin,

- orifice inverse, machine non couplée, remplissage du bassin,

avec des inversions de sens de rotation et démarrages à pleine tension.

Et puisque les marées se manifestent 2 fois par 24 heures, c'est un nombre de démarrages et d'arrêts assez imposant qui est enregistré. 


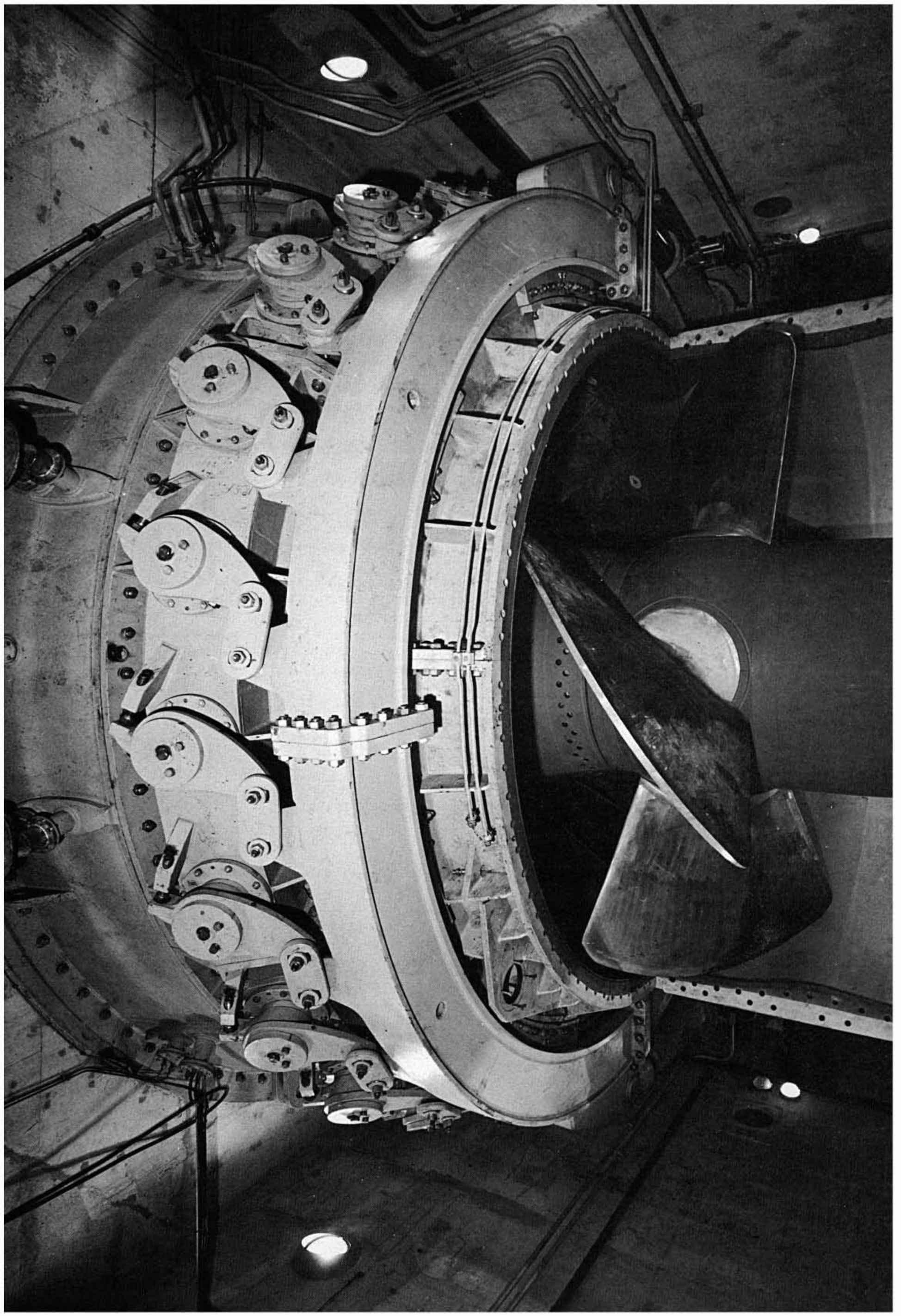


- 4 démarrages journaliers minimum par groupe dans un fonctionnement à double effet :

- ou encore 27500 démarrages annuels pour l'ensemble effectivement réalisés.

Et puisque nous sommes à l'heure des bilans, la centrale a enregistré, depuis sa mise en service 500000 démarrages de groupes.

\subsection{Turbines}

Rappelons tout d'abord que, dans l'ignorance de l'efficacité de la protection cathodique en milieu marin et sur de telles machines, toutes les précautions - peut-être trop ont été prises pour éviter les attaques de la corrosion :

- utilisation d'acier $17 \% \mathrm{Cr}, 4 \% \mathrm{Ni}$, pour 12 jeux de pales, les moyeux de roue, les distributeurs:

- utilisation d'acier $18 \% \mathrm{Cr}, 8 \% \mathrm{Ni}$, pour les manteaux de roue;

- utilisation d'alliage cuivreux, cupro-aluminium pour 12 autres jeux de pales.

Les démontages des machines que nous avons été amené à faire de 1975 à 1982 nous ont permis de constater qu'après environ 130000 heures d'immersion dont 90000 heures de marche, aucune oxydation notable n'avait touché tous ces organes : miracle de la technique, surplus de précautions qui font associer protection cathodique, métaux nobles et peintures?

Probablement l'ensemble de ces trois composantes ajoutées à toutes les recherches et études menées avant la construction de l'ouvrage.

Des travaux d'entretien ont été réalisés au cours de ces révisions qui ont porté sur :

- des recharges en Inoxyda $53 \mathrm{H}$ très localisées en bordure des pales bronze pour éliminer surtout des traces de cavitation,

- des meulages pour éliminer encore des traces de cavitation plus légères sur les pales en acier inoxydable,

- des réfections de portées de joints de pales ont été entreprises par rechargement: sur 24 pales en bronze d'aluminium, et seulement sur 4 pales en acier inoxydable.

Et corrélativement avec changement, sinon obligatoire du moins occasionnel, des joints d'étanchéité, les joints suivants ont été utilisés :

- joint torique caoutchouc, dureté shore 65 sur 44 pales bronze;

- joint chevrons (4 rangées) caoutchouc toilé, dureté shore 70 sur 4 pales bronze et 48 pales en acier inoxydable.

Le démontage des pales a donné quelques soucis sur certains groupes, certaines vis de fixation ont en effet dû être détruites pour en faciliter l'extraction.

Nous pouvions avoir quelques inquiétudes sur le comportement des bagues Celoron dont le gonflement en 1973 avait conduit à un réalésage des pots de directrices. Nous n'avons cette fois constaté aucune dégradation et n'avons pas eu besoin d'augmenter l'alésage.

Au cours des premiers démarrages, quelques incidents s'étaient produits sur le joint radial d'étanchéité : à savoir usure anormale de la $4^{e}$ couronne d'étanchéité. Diverses modifications avaient été apportées (amélioration de l'arrosage et du placage du joint sur la pièce d'usure par une jarretière en caoutchouc). Dès 1974, on a constaté une usure anormale de cette $4^{c}$ couronne. Celle-ci, en carbone, a été remplacée par une couronne de 16 segments en Cestidur qui depuis, n'a pas donné d'inquiétude.

Par contre, au cours des démontages effectués de 1975 à 1982, nous avons constaté une dégradation importante par corrosion de l'ensemble des pièces d'usure du joint. Ces pièces ne sont en effet pas protégées par la protection cathodique.

Quelques dispositions nouvelles ont été prises :

- essai d'une protection cathodique sur 8 groupes mise en place en 80 et 81 ;

- rechargement de certaines pièces d'usure (sous les joints carbones);

- remplacement de 3 pièces d'usure : - une en acier inox $18 / 8,-$ deux en Uranus $55(27 / 6)$ :

- remplacement des 24 pièces de frottement (sous le joint SJMRJT) : - deux en acier $17 / 4$ chromées, -22 en Uranus $55(27 / 6)$;

- recherche de matériaux plus nobles (céramique par exemple).

\subsection{Alternateurs}

\subsubsection{Stator}

Les alternateurs ont été la grosse préoccupation de ces dernières années. Un incident relevé sur le groupe B5 en janvier 1975 - frottement du rotor sur le stator - s'est en effet étendu aux 24 autres machines où, après visites systématiques on a pu constater une réduction quasi générale des entrefers.

C'est donc une campagne de réfection des 24 stators qui a été entreprise et qui, commencée en 1975 ne s'est terminée qu'en mai 1982.

Que s'est-il donc passé ?: les contraintes indiquées au début, les sollicitations permanentes auxquelles sont soumis le matériel n'ont pas été étrangères à cette rupture des clavettes de fixation du circuit magnétique sur la carcasse qu'il a fallu toutes remplacer.

Des dispositions particulières ont alors été prises pour l'organisation de ce chantier pour limiter au maximum les pertes d'énergie, voire de puissance et raccourcir au mieux cette période de mise en état :

- achat de deux batardeaux de coupure supplémentaires; - achat d'une carcasse supplémentaire pour disposer d'un stator réparé d'avance et diminuer le délai d'indisponibilité des groupes;

- " épinglage " de sept groupes pour éviter l'aggravation du phénomène sur les groupes les moins touchés et par suite leur indisponibilité prématurée.

Quelques mots sur cette technique "d'épinglage " qui s'est révélée très efficace :

- perçage de trous dans la carcasse à partir de l'extérieur du bulbe:

- perçage et taraudage des clavettes d'accrochage du circuit magnétique sur la carcasse pour les reprendre par vis et écrous de l'extérieur et les ramener sur la carcasse; - capotage et étanchéité extérieurs pour éviter toute intrusion d'eau de mer. 
Cette opération délicate a demandé 25 jours par groupe et coûté environ $110 \mathrm{KF}$, mais elle a permis de mieux échelonner les répartitions et de garantir une disponibilité de la Rance d'un peu plus de $80 \%$.

Et bien sûr, il a fallu en fin de cycle, reboucher convenablement et étancher ces 4000 trous, fait dans les carcasses dont l'épaisseur est de $77 \mathrm{~mm}$.

\subsubsection{Rotor}

L'usure des pôles par électro-érosion a été le premier défaut constaté sur les rotors et a conduit en 1968 à un démontage complet des 64 pôles des 24 alternateurs (soit dit en passant 1536 pôles à démonter et remonter) pour matage des barres d'amortisseurs dans leur logement et éviter ainsi l'électro-étincelage.

Il n'empêche que, profitant de l'occasion, tous les pôles rotoriques ont été repris pendant la campagne 75-82 et réisolés, les circuits amortisseurs ont été également remis en état avec de délicats problèmes de jonctions interpolaires (machine à souder spécialement conçue pour soudage des connexions).

\subsubsection{Coût de la réparation et incidence sur la pro- duction}

1) Sans trop entrer dans les détails, notons que le coût global de l'opération, en francs 1980, s'est élevé à $4600 \mathrm{KF}$ par groupe, répartis comme suit :

$\begin{array}{lr}\text { - réparation stator ............................... } & 2500 \mathrm{KF} \\ \text { - réparation rotor .......................... } & 300 \mathrm{KF} \\ \text { - révision turbine, démontage, re- } & \\ \text { montage } & \end{array}$

soit pour l'ensemble des 24 machines : $110000 \mathrm{KF}$

Le montant de ces réparations directement répercuté sur le coût de production s'est traduit par une augmentation de 3 centimes par KWH soit environ $17 \%$ de majoration.

2) L'ensemble de cette opération s'est déroulée au rythme moyen de 3 à 3,5 machines par an, chacune d'entre elle étant immobilisée environ 9 mois.

La disponibilité de la Centrale a varié pendant cette période de 70 à $94 \%$ et les pertes d'énergie moyennes annuelles pendant ces 7 années ont été de $100 \mathrm{GWh}$.

\subsection{Vannes}

Précisons tout de suite que lorsqu'on parle de vannes à la Rance, il s'agit des 6 vannes des pertuis de vidange et de remplissage accélérés du bassin, soit 6 vannes de $15 \mathrm{~m} \times 10 \mathrm{~m}$, commandées par des servomoteurs à huile et sur lesquelles passe le pont routier, objet de nos litiges avec le Conseil Général d'Ille-et-Vilaine.

Si de 1968 à 1972, sans protection cathodique le comportement des rails de roulement en acier $17 / 4$ a été satisfaisant, il n'en a pas été de même des fusées des galets de roulement qu'il a fallu reprendre. Le tablier des vannes, en acier ordinaire, qui était seulement peint se comportait d'une manière satisfaisante mais nul doute, que la peinture partie, nous nous serions trouvés devant un problème d'oxydation aigü.

Une protection cathodique a donc été mise en place et après peinture systématique des 6 vannes, les traces d'oxydation n'ont été depuis que finalement très légères.
La conception de ces vannes avec un minimum d'angles vifs a beaucoup contribué à leur excellent état, mais il est néanmoins nécessaire de les surveiller et de les peindre de temps en temps. C'est ainsi qu'une nouvelle campagne de peinture a été lancée en 1979 et que 3 vannes ont déjà été repeintes.

Quelques précisions techniques qui pourraient intéresser les spécialistes :

Après un sablage très soigné :

- application d'une couche de Wash Primer de 8 à 10 microns,

- application de deux couches de primaire, de couleur différente verte et jaune pour contrôler la bonne mise en place, épaisseur 100 microns,

- application d'une couche antifouling vinylique de 75 microns,

soit une épaisseur finale de 185 microns.

Ces opérations de peinture d'une vanne nécessitent tout de même le batardage de la passe. L'indisponibilité est alors de 6 mois, pour une faible pente d'énergie qui peut être évaluée à GWh et un coût de $600 \mathrm{KF}$, francs 1982 .

L'utilisation de la boulonnerie cadmiée, le chromage des tiges des servomoteurs éliminent pratiquement tout autre entretien sur ces ouvrages.

On considère que c'est à une fréquence de 13 à 15 ans que ces vannes doivent être reprises. Il suffit quand même d'imaginer les quelques embruns que peut engendrer ce torrent de $6000 \mathrm{~m}^{3} / \mathrm{s}$ qui 4 fois par jour passe sous ces vannes pour penser que ces protections associées, peinture et protection cathodique, ne sont pas tout à fait superflues.

Quelques notes maintenant sur les vannes de coupure au nombre de 8 , qui permettent la mise hors d'eau d'un bulbe par condamnation et vidange du pertuis. Elles sont aussi l'objet de nos sollicitudes, sont peintes avec les mêmes exigences que les précédentes mais dans des conditions bien meilleures puisque nous disposons de 2 salles de peinture protégées de l'eau et des intempéries qui garantissent de bonnes conditions d'applications et de séchage.

Elles ont été toutes peintes de 1972 à 1974 et ont été remises en état en 1982 et 1983 après les nombreuses manipulations de ces dernières années qui ne se sont pas faites sans quelques égratignures.

\subsection{Matériel électrique}

L'isolation de tout le matérial électrique du milieu agressif qu'est l'ambiance marine, le prélèvement d'air de ventilation le plus loin possible de l'estuaire a préservé l'appareillage électrique de toute corrosion et nous n'avons eu aucun incident qui pourrait être dû au qualificatif " marémotrice " de l'installation. Si certains matériels ont vieilli, ils l'ont fait aussi bien qu'ailleurs, et ils ont été ou seront remplacés sans qu'on puisse dire qu'ils auraient vécu plus ou moins longtemps dans une autre Centrale.

C'est ainsi que la pièce maîtresse de l'installation, le calculateur de conduite doit être remplacé en 1985. Il a rendu et rend encore aujourd'hui tous les services qu'on attendait de lui, s'il est un peu dépassé maintenant c'est que nos exigences sont autres en 1984, mais aussi qu'il devient de plus en plus difficile d'en assurer la maintenance et de se procurer des pièces de rechange. 
Le renouvellement du calculateur conduira, nous l'espérons, à une exploitation automatique et c'est ainsi que, dans quelques années, nous n'aurons plus à la Rance qu'un seul Service de Quart, celui de l'écluse, où la présence d'un agent reste indispensable compte-tenu de l'environnement : voies routières et navigables, visiteurs...

\subsection{Autres matériels}

\subsubsection{Tuyauteries}

Les tuyauteries ont été un sujet préoccupant posé aux constructeurs de la Rance, elles ont fait l'objet d'études très sérieuses elles aussi. Toutes celles où transite l'eau de mer sont en acier inoxydable 17/4 ou éventuellement en PVC et n'ont pas donné lieu depuis la mise en service, à de préoccupations particulières, si ce n'est une exception de taille, la conduite de vidange, tuyau de $\bullet 350 \mathrm{~mm}$ et 350 $\mathrm{m}$ de longueur qui, tout au long de la Centrale recueille à la côte $-12 \mathrm{~m}$, les eaux de fuite dont on a dit qu'elles n'étaient que de $51 / \mathrm{mn}$, mais aussi les eaux de vidange des pertuis quand il s'agit d'isoler un groupe pour une visite extérieure. Cette tuyauterie a présenté beaucoup de défectuosités, particulièrement aux soudures et après la pose d'une vingtaine de,colliers de sécurité, nous avons dû nous résoudre à son remplacement total par une conduite en stratifié de fibres de verre imprégnées de résine époxy de diamètre o 300 et de $7,5 \mathrm{~mm}$ d'épaisseur, opération qui a coûté $1000 \mathrm{KF}$ mais qui maintenant devrait garantir un service sans problème pendant de nombreuses années.

\subsubsection{Pompes d'exhaure}

16 pompes d'exhaure - un grand nombre pour de si petites fuites, mais nous jouons la sécurité avant tout - assurent donc l'évacuation des eaux résiduelles (vidanges, fuites). Essentiellement en bronze d'aluminium, elles ne présentent pour ainsi dire pas d'attaque par corrosion, mais restent très sollicitées par l'abrasion, et aussi la cavitation... La remise en état des pompes n'est pas un problème en soi, elle reste toutefois une opération longue et coûteuse eu égard au matériel en cause : remise en état de roues et labyrinthes en bronze, pas toujours facile à recharger, et de petites dimensions, longueur de la ligne d'arbre $(14 \mathrm{~m})$. La solution à terme est probablement leur remplacement par des pompes immergées, courantes sur le marché, bien qu'il s'agisse de matériel inoxydable et nous nous en préoccupons maintenant.

\subsubsection{Filet de protection des navigateurs}

En 1973, un dispositif de protection des navigateurs demandé à l'Établissement par l'Administration a été mis en place, à l'amont et à l'aval du barrage. Ce que nous avons pris l'habitude d'appeler un "filet ", n'est finalement qu'une ligne de bouées enfilées sur un câble d'acier inox ou galvanisé et surmontée d'une saisine, laquelle permet aux navigateurs imprudents de s'accrocher sinon d'attendre un éventuel secours.

La mise en œuvre de cette protection a été laborieuse dans la mesure où les courants en Rance, au droit des vannes par exemple sont très forts, que les encrages doivent en conséquence tenir des efforts importants, 13 tonnes, et que le Service des Phares et Balises qui n'a pas l'habitude de calculer et d'implanter de tels ouvrages ne nous a pas été d'une grande aide, alors que nous comptions sur lui pour nous déterminer la forme et le volume des bouées (dont certaines ont un volume de $13 \mathrm{~m}^{3}$ ).

L'achat, la mise en œuvre de cette protection, $3000 \mathrm{~m}$ de long et 2500 bouées support pour 9 bouées d'ancrage, a coûté environ $3000 \mathrm{KF}$ de 1973 à 1976. Les charges d'entretien ont été très lourdes les premières années, accrues par des difficultés inconnues (tenue des orins et ancrages, des bouées, des câbles...) elles ont atteint $1000 \mathrm{KF}$ en 1981. Des études menées au Laboratoire de Mécanique de Nantes et avec différents Constructeurs pour une bonne qualification de bouées et autres matériels ont permis de réduire au minimum ces charges et c'est ainsi qu'en 1982, elles n'étaient plus que de $350 \mathrm{KF}$.

Si ce "filet " a mobilisé pas mal d'heures et nécessité beaucoup de matière grise, il a tout de même eu l'avantage de retenir quelques imprudents : plaisanciers ignorants des règles de la mer qui s'aventurent dans une zone interdite déjà matérialisée par des bouées conventionnelles aux couleurs internationales, véliplanchistes débutants qui, au gré des vents et courants ne peuvent plus ramener leur frêle esquif et qui tous, finissent accrochés à ce dernier secours et c'est l'un d'eux qui, épuisé, a tout lâché et est passé sous une des grandes vannes pour finir, pour le moins étourdi, sur les rochers de la rive droite à l'amont du barrage, c'était en 1982.

\subsection{Protection cathodique}

On ne peut parler de la Rance sans évoquer la protection cathodique. Monsieur Lambert l'a parfaitement décrite et exposée en ces lieux, en d'autre temps. Il nous paraît intéressant de rappeler sommairement ce qu'elle est, pour insister, une fois encore sur les résultats positifs qui ont été enregistrés :

la protection cathodique des machines comporte pour chaque groupe 3 couronnes de 12 anodes en tentale platiné à 50 microns,

- une couronne sur le manteau de roue côté mer;

- une couronne sur le distributeur;

- une couronne sur l'anneau à tirants (8) et le bras d'accès (4).

Celle des vannes est assurée par 4 tubes en titane platiné à 5 microns par vanne.

Celle de l'écluse par 16 tubes également en titane platiné.

Toutes ces anodes sont alimentées par 3 redresseurs sous une tension de $10 \mathrm{~V}$, un courant de $20 \mathrm{~A}$ et la consommation annuelle est de $150000 \mathrm{KWh}$.

L'examen de tous les ouvrages protégés montre l'efficacité de cette protection et la confirmation du "bon choix " fait lors de la construction et si la protection des vannes n'a été réalisée que quelques années plus tard, il faut noter que son installation a été plus difficile et qu'à tout prendre il vaut mieux, quitte à faire du surabondant, prévoir un peu plus à l'origine que de faire, par la suite, des adjonctions dans des conditions difficiles.

D'autre part, nous avons partout constaté la présence d'un dépôt calcomagnésien de quelques microns sur toutes les parties non peintes qui, si on le gratte, laisse voir un métal sain et brillant toujours sans trace d'oxydation, il devient donc, lui aussi un organe de protection supplémentaire. 


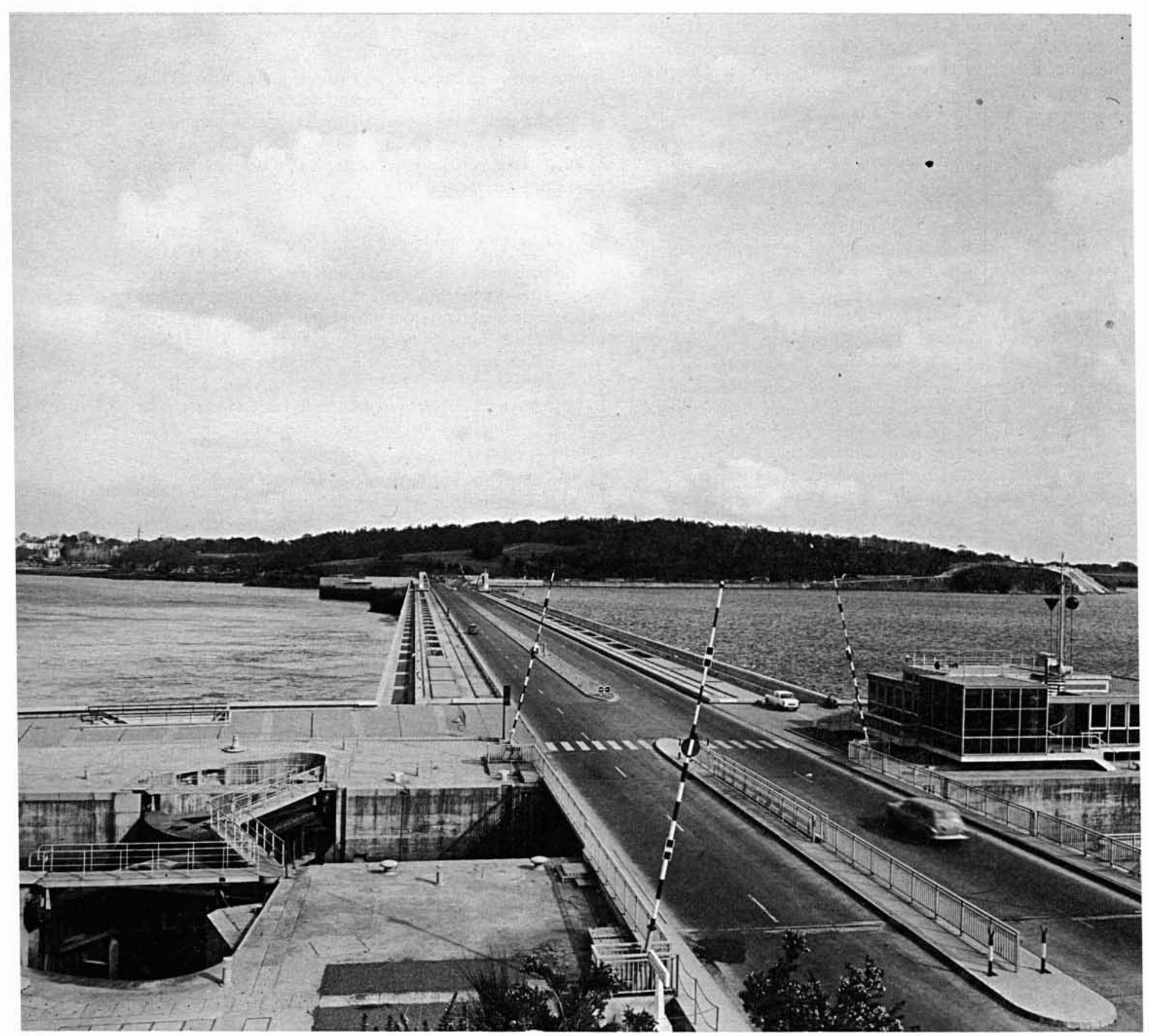

Photothèque EDF 
Il est évident que, compte-tenu des résultats et des constatations faites, la protection cathodique est l'objet de toute notre attention et de notre surveillance, qui se traduisent par une maintenance, tout compte fait non négligeable; soit par an 9500 mesures de tension ou de courant, bimensuelles, trimestrielles ou annuelles.

Une partie de ces mesures pourraient être prises en charge par le nouveau calculateur en 1985.
Nous voudrions ajouter également à ce sujet, que la "Nova Scotia Power Corporation" au vu des résultats obtenus à la Rance et qui installe, en baie de Fundy sur l'Annapolis River un groupe immergé a fait appel à Gaz de France pour l'installation d'une protection cathodique.

Cette nouvelle expérimentation pourrait être le prélude de la seconde centrale marémotrice au Monde.

\section{Résultats d'exploitation}

\subsection{Production}

Les études précédant la mise en exploitation de la Rance avaient conduit aux prévisions suivantes :

- turbinage

$608,5 \mathrm{GWh}$

- pompage

64,5 GWh

- production nette

544 GWh

Qu'en est-il de ces prévisions, 20 ans ?

La centrale de la Rance a produit depuis sa mise en service et à fin 1983: $8500 \mathrm{GWh}$ et a consommé une énergie de pompage de $700 \mathrm{GWh}$, ce qui dégage une production nette de : $7800 \mathrm{GWh}$

Quelques grandes périodes peuvent être dégagées :

\section{a) de la mise en service à fin 1968}

La production n'est pas significative, puisqu'il s'agit d'une période d'essais de démarrages et de mises au point. A fin 1968 , la disponibilité n'est que de $77 \%$ et la production annuelle brute de $426 \mathrm{GWh}$.

\section{b) de 1969 à 1974}

Période d'exploitation normale avec recherche des meilleurs fonctionnements et qui, après les premiers balbutiements tend vers une modulation moindre des coûts pour parvenir à une gestion à optimum énergétique, c'est-à-dire à des coûts constants.

La disponibilité de la centrale est très satisfaisante, varie de $86 \%$ en 1969 pour atteindre $95,5 \%$ en 1974, la production brute atteint même un maximum de $606 \mathrm{GWh}$ à fin 1974, c'est la période euphorique, les courbes atteignent les sommets, la Rance concrétise, presque, tous les espoirs mis en elle (la production nette n'est que de $506 \mathrm{GWh}$ car le pompage a consommé cette année là $90 \mathrm{GWh})$.

\section{c) de 1975 à 1982}

Surviennent en 1975 les graves incidents sur les alternateurs. La disponibilité de la centrale tombe à $71 \%$ en 1976 , mais malgré cela, et compte-tenu des dispositions prises dont on a parlé précédemment ajoutées à l'interruption du pompage de 1976 à 1980 , la production se maintiendra au cours de cette période à un niveau correct.

Elle sera de $444 \mathrm{GWh}$ en 1976, mais atteindra déjà $607 \mathrm{GWh}$ en 1982 et battra là une première fois son record annuel, malgré une disponibilité qui n'est que de $94 \%$ et une diminution d'amplitude des marées de $3 \%$ entre 1979 et 1982 .

\section{d) de 1983 à... plus tard}

La production 1983 est la production record : $609 \mathrm{GWh}$, mais les $97 \mathrm{GWh}$ de pompage atténuent le solde net.

- malgré un changement d'exploitation fonctionnement à recette maximale avec un coût d'énergie qui varie de 1 à 5 (soit 7 à 35 centimes);

- malgré encore une diminution d'amplitude des marées qui a été de $0,7 \%$ par rapport à 1982 ;

- mais aussi grâce au nouveau programme AGRA mis en application depuis 1982.

Toutefois, les records de production ne veulent plus dire grand-chose quand tant de paramètres, qui eux ne sont pas orientés vers ce maximum, interviennent. Tous ces records n'ont plus maintenant qu'une valeur relative et il n'y a pas lieu de s'en plaindre. Les Hydrauliciens que nous sommes pourraient en être choqués, les Economistes que nous devenons ne doivent que s'en féliciter.

\subsection{Pertes d'énergie}

Elles sont dues en régime de croisière à des indisponibilités nécessaires aux visites systématiques et aux contrôles des machines, elles sont en année normale de l'ordre de $5 \mathrm{GWh}$, soit $1 \%$ de la production.

Nous nous efforçons d'avoir les 24 groupes disponibles pendant les semaines de fortes marées et de faire nos entretiens en marée de mortes eaux (à titre indicatif, les rapports de production entre une marée de morte eau et une marée d'équinoxe sont dans le rapport de 1 à 18 !).

\subsection{La Rance dans l'Ouest}

La Rance intervient en Bretagne pour $8 \%$ de la consommation des quatre départements bretons, mais pourrait subvenir à $30 \%$ de la consommation de son département : l'Ille et Vilaine.

Elle représente encore $15 \%$ de la puissance appelée sur la Bretagne, malheureusement pas toujours aux moments les plus favorables.

\subsection{Coût d'exploitation}

La Rance est exploitée par 60 agents répartis en différentes équipes d'exploitation et d'entretien électromécanique. Les dépenses annuelles correspondantes sont de l'ordre de $30000 \mathrm{KF}$ et le prix du $\mathrm{kWh}$ produit était, en 1982, toutes charges comprises de 16,9 centimes. 


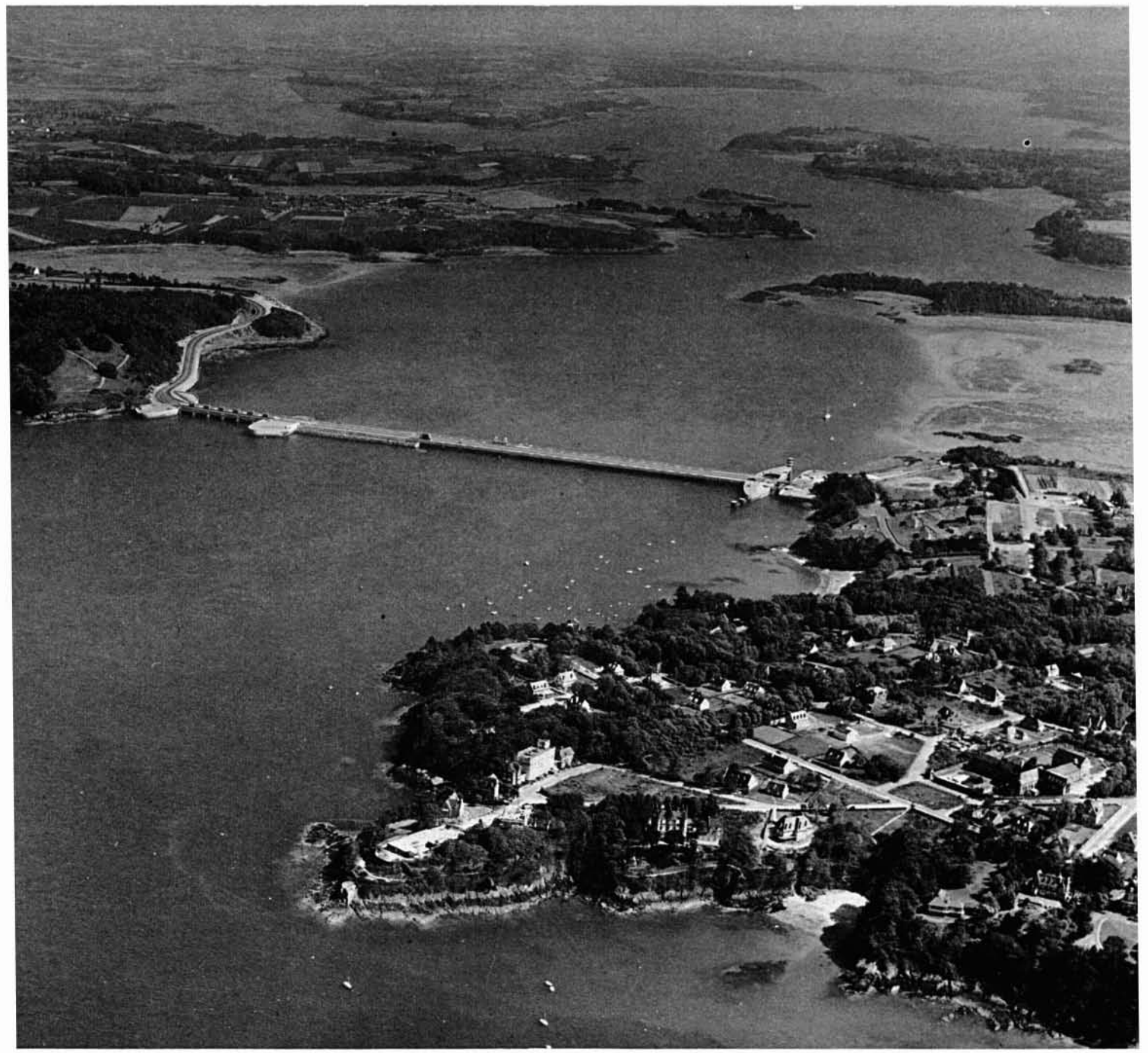




\section{Environnement}

Et maintenant, s'il vous arrive de passer sur le barrage de la Rance, de flâner en regardant la tour Solidor et de constater que l'étale côté bassin est un peu plus longue qu'habituellement, pensez seulement que les mouvements d'énergie auront programmé le démarrage de la centrale quelques dizaines de minutes plus tard, pour satisfaire un besoin... énergétique ou gagner quelque argent supplémentaire. Pensez aussi que ces longues marées, ces modifications apportées à un cycle naturel, immobile pendant des millénaires ont perturbé un environnement habitué à tout autre chose, environnement qui nous est cher, pas toujours, mais qui risque, un jour, de nous coûter très cher.

L'environnement de la Rance n'a pas fait l'objet, lui, en 1960, d'études particulières. C'est maintenant une suite de sujétions, une succession de contraintes, de réclamations, de problèmes.

Ce pourrait être un pont magnifique lancé entre St. Malo et Dinard qui raccourcit les trajets d'une quarantaine de kilomètres entre les deux cités, ce pourrait être ce havre de tranquilité offert aux 18000 plaisanciers qui franchissent annuellement l'écluse, pas toujours dans le calme et la bonne humeur, ce pourrait être encore cet objet touristique qui reçoit bon an, mal an, une vingtaine de milliers de visiteurs venus du Monde entier, mais c'est encore tout autre chose.

\subsection{Problèmes écologiques}

Le Laboratoire maritime du Museum national d'histoire naturelle de Dinard a étudié avec soin les conséquences de l'implantation du barrage sur l'environnement biologique. Nous reprendrons ses conclusions :

«En transformant le cycle des marées naturelles - diminution de l'amplitude moyenne, prolongation des étales -, l'usine marémotrice a provoqué une série de modifications qui ont profondément remanié le milieu :

- les niveaux du bassin sont soumis à un régime artificiel et irrégulier;

- l'amplitude des marées entraîne entre $10,5 \mathrm{~m}$ et $11 \mathrm{~m}$ un allongement de la durée d'immersion;

- le relèvement du niveau moyen fait reculer vers l'amont la zone d'influence des décharges d'eau douce provenant de la rivière;
- les modifications de la dynamique des courants s'accompagnent d'une redistribution sédimentaire sans que l'on puisse dire que la présence du barrage ait accéléré l'envasement en Rance.

Aussi, a-t-on en Rance maintenant un bassin marin véritable petite mer intérieure entre le barrage et Port St. Jean et un estuaire, confiné dans le fond à l'amont de Port St. Jean.

Les conséquences biologiques sont plus difficiles à cerner cependant :

- l'isolement quasi total du bassin pendant la construction de 1963 à 1966 a provoqué la disparition presque complète des peuplements originels;

- la mise en service à partir de 1967 a permis l'installation progressive de peuplements normaux et diversifiés et pêcheurs et plaisanciers continuent à trouver en Rance, bars, mulets, anguilles, homards, ormeaux, seiches...

De toute manière, il semble fondamental de rappeler que la stabilité écologique du système et le niveau de sa production sont étroitement liés à la régularité des conditions d'exploitation de la Rance. - Et c'est pourquoi, en accord avec le Service des mouvements d'énergie, nous nous efforçons, au détriment parfois de la rentabilité, de modifier des programmes qui, par exemple, conduisent à une étale de $24 \mathrm{~h}$ un dimanche, et éviter ainsi ces déphasages nuisibles à cette faune ou flore microscopique qui peuplent l'estuaire qu'on connait bien sous le nom de " plancton " mais qu'on imagine mal quand on nous parle de "Pelvetia Canaliculata " ! -

\subsection{Problèmes humains}

Ce sont ces pêcheurs, ces ostréiculteurs qui ne pêchent "plus comme avant" en Rance. Ce sont aussi ces plaisanciers qui ne trouvent plus un chenal qu'indiquent encore les cartes marines, ce sont encore tous ces riverains dont le paysage a été changé et qui ne retrouvent plus leur Rance et c'est enfin, après ce long état des lieux de notre centrale, l'anecdote finale : ces sprinters d'une course à vélos qui, un jour de juillet, trouvent en face d'eux les deux immenses ponts levés pour laisser passez de beaux et paisibles voiliers.

\section{Conclusion}

Alors, en conclusion, que penser de cet ouvrage ?

S'il fallait maintenant prendre la décision de le construire, il est probable que face aux grands chantiers nucléaires actuels, les $500 \mathrm{GWh}$ ne pèseraient pas lourd dans la balance pour une décision favorable et c'est peut-être pourquoi la Rance est encore la seule usine marémotrice construite sur la planète.

Quoiqu'il en soit, nous disposons d'un outil de travail intéressant, performant et compte-tenu de ce que nous savons de lui, nous espérons le conserver longtemps et en excellent état. 


\section{Discussion}

Président : M.R. VULSER

M. le Président remercie M. Hillairet en insistant sur le fait que le fonctionnement de l'usine est bien maîtrisé.

A une question de M. Poirel, M. Banal rappelle laspect périodiques des variations d'amplitude des marées ( $\approx 18$ ans $2 / 3$ ). On est dans la phase où l'amplitude diminue après avoir été moyenne en 1983. M. BANAL précise aussi que les statistiques E.D.F. présentent la production réelle; pour comparer à la plupart des autres pays, il conviendrait d'y ajouter la consommation moyenne des auxiliaires - soit $8 \mathrm{GWh}$ par an.

M. le Président et $\mathrm{M}$. le rapporteur insistent sur l'exploitation qui est conduite à rentabilité économique maximale avec beaucoup de déplacements de production (et non à production maximale).

M. BOURDON. - "l'expérience de la Rance, combinée avec celle des hélices propulsives, permettrait-elle de choisir entre pales en acier inoxydable et pales en cupro-aluminium ?". Cette question provoque des réponses de MM. Hillairet, BANAL,
PoIrel et Bosc, d'où il ressort que les expériences sur les hélices ne sont pas tout à fait comparables car d'un côté les périodes d'entretien sont plus fréquentes et pour des raisons de caractéristiques mécaniques, de résistance à l'usure et de facilité d'intervention, on préfèrerait sans doute l'acier inoxydable; mais le comportement des deux matériaux est très satisfaisant, l'écart technique est faible, qui pourrait être modifié par des considérations économiques.

M. LEFOULON pose la question de la taille unitaire limite actuelle. M. Bosc: "Les études de faisabilité ont été faites jusqu'à $100 \mathrm{MW}$, mais il faut tenir compte du débit et de la hauteur de chute ".

M. ROBERT croît utile de rappeler les " retombées " techniques des groupes bulbe de La Rance. L'expérience ainsi acquise a été utilisée sur le Rhin et sur le Rhône. L'emploi de turbines à axe horizontal a permis "si je ne me trompe", des économies d'équipement de l'ordre de $30 \%$ pour le génie civil des usines. 